\title{
Serial Measurements of Fetal Head Circumference and Abdominal Circumference to Predict Fetal Growth Restriction in a Sri Lankan Study Population
}

\author{
Rasika S de Silva ${ }^{1}$, Hemantha Perera ${ }^{2}$
}

\begin{abstract}
Aim and objective: Prediction of fetal growth restriction (FGR) by serial ultrasound measurement of head circumference (HC) and abdominal circumference (AC) of the fetus applied routinely to all mothers irrespective of risk status for FGR and small for gestational age.

Materials and methods: A prospective study was done of 508 pregnant women who underwent two successive growth scans 4 weeks apart at Sri Jayewardenepura General Hospital, Sri Lanka. FGR was identified by graphically plotting serial fetal AC and HC. Postnatally, growth restriction was diagnosed based on ponderal index (PI). Sensitivity, specificity, positive predictive value, and likelihood ratio of predicting FGR by successive serial ultrasound measurements of fetal $A C$ and $\mathrm{HC}$ were calculated.

Results: Based on fetal AC and HC, FGR was present in 223 of 508 fetuses (43.89\%). Based on PI, 224 of 508 (44.1\%) neonates were growthrestricted. Sensitivity, specificity, positive predictive value, positive likelihood ratio, and negative likelihood ratio of predicting FGR by serial fetal AC and $\mathrm{HC}$ were $82.59,86.62,82.59 \%, 6.2$, and 0.2 , respectively.

Conclusion: Serial ultrasound measurements of fetal AC and HC plotted on a fetal growth centile chart routinely carried out in all mothers irrespective of risk status for FGR increases the detection of FGR.

Keywords: Fetal growth restriction, Noncommunicable diseases, Ponderal index, Serial ultrasound scans, Thrifty phenotype.

Journal of South Asian Federation of Obstetrics and Gynaecology (2021): 10.5005/jp-journals-10006-1933
\end{abstract}

\section{SYNOPSIS}

Detection of fetal growth restriction irrespective of risk status is paramount to prevent unexplained intrauterine fetal deaths (IUFD) and adult noncommunicable diseases.

\section{INTRODUCTION}

About $60-80 \%$ of the four million neonatal deaths that occur worldwide every year are associated with low birthweight (LBW) caused by fetal growth restriction (FGR) or preterm delivery or both.' Majority of FGR results from placental dysfunction which affects fetal nutrition and oxygen supply. Antenatal detection of FGR and optimized delivery would significantly reduce related perinatal morbidity and mortality.

FGR is defined as an inability to achieve the genetic growth potential by the fetus. ${ }^{2,3}$ The causes of FGR can be categorized as maternal, fetal, and placental. In the majority of cases, reduced oxygen transfer to the fetus across the placenta is the underlying cause. Placental insufficiency is thought to be a result of improper invasion of maternal spiral arterioles by extra villous trophoblast cells. Leading maternal causes include medical disorders, infectious diseases, malnutrition, and smoking. Main fetal causes include fetal congenital anomaly and fetal infection.

Small for gestational age (SGA) is often synonymously and erroneously used with FGR because most of the immediate complications after birth are related to LBW irrespective of the underlying reason for LBW. ${ }^{4,5}$ However, following the "thrifty phenotype" hypothesis, this approach has to be reconsidered in view of the long-term medical complications which have been found to be related to FGR irrespective of the birthweight. ${ }^{6-9}$ SGA includes some fetuses that are genetically determined to be
${ }^{1}$ Department of Maternity, Dudley Group of NHS Foundation Trust, Dudley, United Kingdom

${ }^{2}$ Department of Obstetrics and Gynaecology, Sri Jayewardenepura General Hospital, Kotte, Sri Lanka

Corresponding Author: Rasika S de Silva, Department of Maternity, Dudley Group of NHS Foundation Trust, Dudley, United Kingdom, Phone: +07448260875, e-mail: rasikasagara@yahoo.com

How to cite this article: de Silva RS, Perera H. Serial Measurements of Fetal Head Circumference and Abdominal Circumference to Predict Fetal Growth Restriction in a Sri Lankan Study Population. J South Asian Feder Obst Gynae 2021;13(4):202-206.

Source of support: Nil

Conflict of interest: None

constitutionally small. The growth trajectory of these fetuses is normal. They are not growth-restricted, but just physically small (SGA without FGR). However, some fetuses in the SGA group could actually be ones who could not reach their genetically determined weight, thus becoming SGA. These fetuses are the ones actually growth-restricted (SGA with FGR). This should now point to another group of fetuses whose weight is actually above the 10th centile but who have not reached their growth potential. They are not SGA but are nevertheless growth-restricted (FGR without SGA). The last group of course would be fetuses who are neither growth-restricted nor below the 10th centile (no FGR and no SGA). Hence, it should be clear that birthweight or estimated fetal weight (EFW) alone could not be used to detect true FGR.

FGR could be symmetrical or asymmetrical. Symmetrical FGR which is less common and usually begins in early gestation. 
Asymmetrical FGR is more common and is detected at later gestational ages. Here, the fetal head continues to grow at normal or near-normal rates (head sparing effect) while fetal abdominal growth trajectory lags behind. However, persistent severe placental insufficiency may eventually lead to restriction of the growth of the head as well.

Late diagnosis of FGR may be hazardous to the fetus since gradually reducing nutrients and oxygen supply may compromise development of organ systems of the body. It may eventually cause intrauterine fetal deaths (IUFDs). The thrifty phenotype hypothesis describes that reduced fetal growth is strongly associated with a number of chronic adult diseases due to fetal adaptations to survive in an environment which has limited availability of nutrients. ${ }^{6-8}$ The said adaptations can be permanent and could lead to chronic medical disorders. ${ }^{9}$ Therefore, it is paramount that a sensitive method is developed to correctly diagnose FGR to minimize perinatal morbidity, mortality, and adult disease.

Plateauing of growth trajectory plotted on fetal growth charts and umbilical artery Doppler waveform abnormalities are frequently used in the diagnosis of FGR worldwide. ${ }^{2}$ Fetal growth charts utilizing ultrasonic fetal biometry parameters have been developed to monitor fetal growth. ${ }^{10,11}$ Customized fetal growth charts, taking into consideration of maternal variables, have been introduced to increase the accuracy of FGR detection. ${ }^{12,13}$ WHO global survey of fetal weight standards has been adopted to Sri Lanka with the aim of detecting the trend of fetal growth. ${ }^{14}$ Ponderal index (PI) is a neonatal counterpart of body mass index, illustrates the degree of nourishment, and could be used to confirm the diagnosis of FGR after delivery. ${ }^{12,15-23} \mathrm{PI}$ is calculated by birthweight divided by the cube of the birth length (weight/length ${ }^{3}$ ). Babies, who are confirmed postnatally to have had FGR in utero, have to be followed up with a tailored plan as they are vulnerable to adult disease irrespective of the birthweight.

\section{Materials and Methods}

A prospective cohort study was done including a cohort of 508 pregnant mothers attending the antenatal clinic of ward 9, Sri Jayewardenepura General Hospital, Sri Lanka. Ethical approval was obtained from the ethics review committee of the hospital prior to the study. Criteria for inclusion for the study were as follows: mothers with a singleton pregnancy, having an accurate dating scan prior to 20 weeks and mothers who gave their written consent for the study. Those who had booked later than 30 weeks and who had medical disorders complicating their pregnancy were excluded from the study. Recruitment started in August 2013 and was completed in March 2014. All were followed up until delivery without dropouts. All subjects had two serial ultrasound scans. First scan was carried out between 24 and 30 weeks, and a second scan after 2-4 weeks from the first scan. Measurements of biparietal diameter (BPD), head circumference (HC), abdominal circumference $(A C)$, femur length ( $F L)$, amniotic fluid index, umbilical artery Doppler resistance index, and pulsatility index were recorded. AC and $\mathrm{HC}$ were plotted on the growth chart developed by Loughna et al. ${ }^{10}$ as Sri Lanka is yet to develop its own population-based AC and HC standard nomograms. A visual assessment, as would be done in a clinic setting, was carried out after the second scan to determine whether the trajectory lines for both $\mathrm{HC}$ and $\mathrm{AC}$ followed the expected centile line on the chart.

The mothers whose trajectory line for AC alone or both AC and $\mathrm{HC}$ deviated to the right of the original centile line were assigned to the "antenatally growth-restricted" group, whose trajectory line for $\mathrm{AC}$ alone or both $\mathrm{AC}$ and $\mathrm{HC}$ followed the original centile line or deviated to its left were assigned to the "antenatally non-growthrestricted" group.

All were followed up and were delivered in the same unit of Sri Jayewardenepura General Hospital during November 2013-June 2014. Weight and length of neonates were recorded soon after the delivery, and PI was calculated using the following formula:

$$
\mathrm{PI}=\text { Birthweight }(\mathrm{kg}) / \text { Length }(\mathrm{m})^{3}
$$

The neonates with a PI below fifth centile in Landmann's PI centile chart, ${ }^{24}$ were assigned to the "confirmed growth-restricted newborn" group. The neonates whose PI was on or above the fifth centile were assigned to the "confirmed non-growth-restricted newborn" group. Sensitivity and specificity of serial ultrasound measurements of fetal $\mathrm{HC}$ and $\mathrm{AC}$ to detect antenatal FGR were calculated.

\section{Results}

The range of age was 18-41 years with an average age of 29.9 years. Median age was 30 years. Standard deviation was 4.57 years. Figure 1 shows the distribution of age.

Out of 508 mothers, 243 (47.83\%) were primigravidae. Distribution of parity is shown in Table 1.

Out of 508 deliveries, 256 (50.3\%) were vaginal deliveries and 252 (49.6\%) were caesarean sections for varied indications. Period of amenorrhea (POA) at delivery ranged from $33+1$ to 41 weeks. Average POA was $37+5$ days with a standard deviation of 8.1 days. Figure 2 shows the distribution of POA at delivery.

About 223 neonates out of 508 (43.89\%) were found to be in the "antenatal growth-restricted" group and 285 (56.11\%) qualified to be in the "antenatal non-growth-restricted" group. Distribution

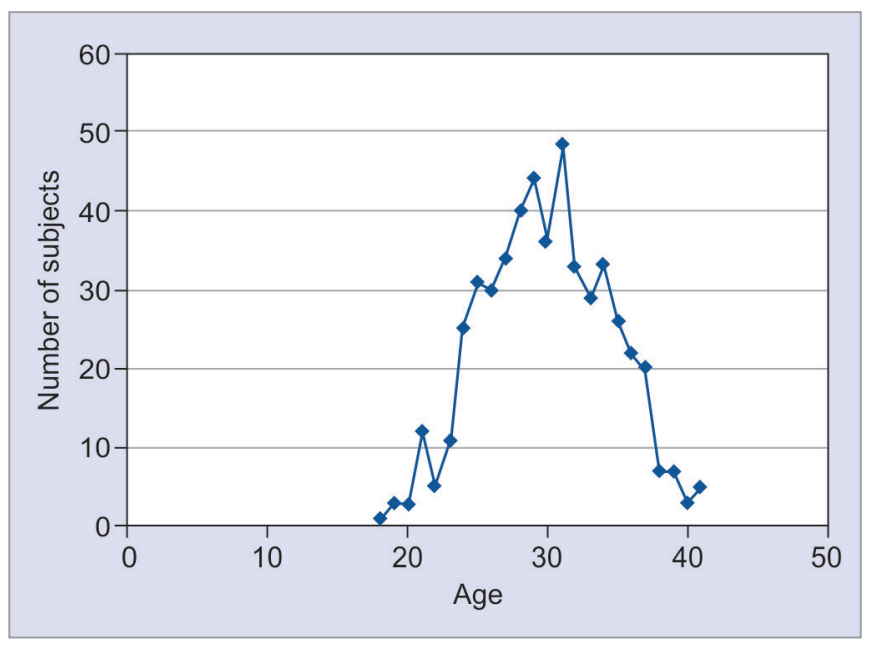

Fig. 1: Distribution of age

Table 1: Distribution of parity

\begin{tabular}{lcc}
\hline Parity & Number & Percentage \\
\hline 0 & 243 & 47.83 \\
1 & 198 & 38.97 \\
2 & 51 & 10.03 \\
3 & 16 & 3.15 \\
Total & 508 & 100 \\
\hline
\end{tabular}


plots of lines connecting two scans for AC of growth-restricted cases are shown in Figure 3. Same plot of non-growth-restricted cases is shown in Figure 4.

Two hundred and twenty four (44.1\%) neonates were assigned to the "confirmed growth-restricted newborn" group, and 284 (59.9\%) neonates were assigned to the "confirmed non-growthrestricted newborn" group. We considered postnatal detection of growth restriction by $\mathrm{PI}$ as true growth restriction and calculated the sensitivity of predicting FGR by serial ultrasound measurements of fetal HC and fetal AC. Thus, 185 out of 223 in the "antenatal

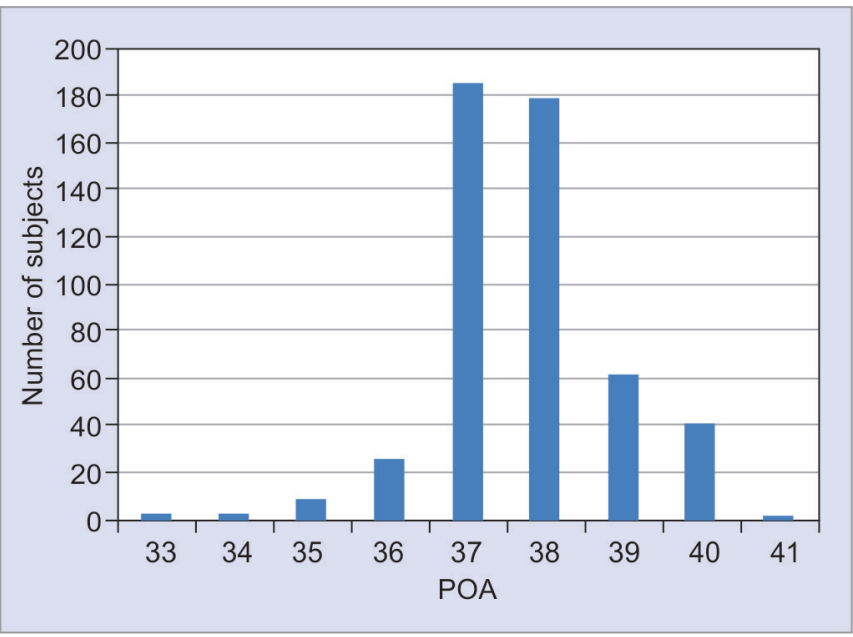

Fig. 2: Distribution of gestational age at delivery

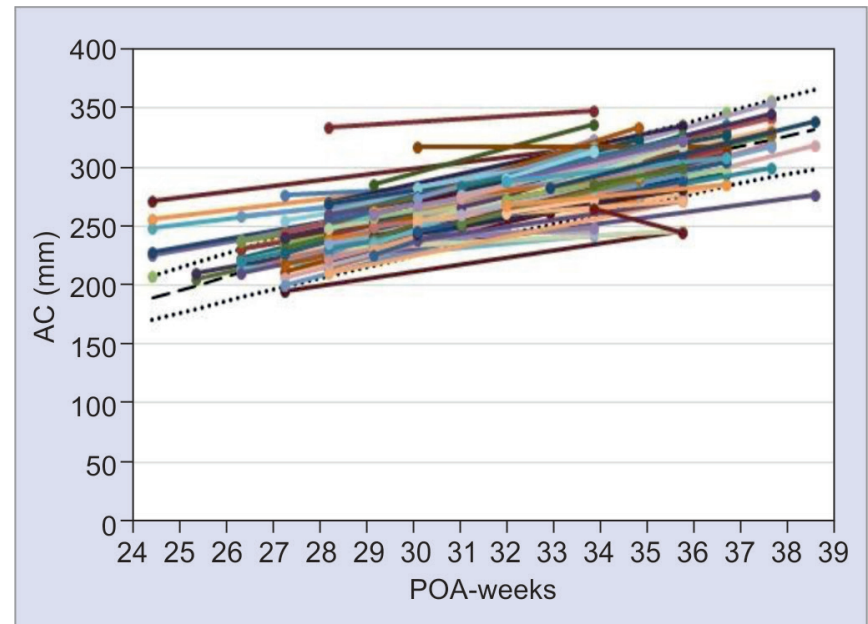

Fig. 3: Distribution of lines connecting two scans of AC in growthrestricted fetuses. Dotted lines represent centile of the nomogram growth-restricted" group found to have growth restriction postnatally (PPV $=82.95 \%$ ). About 246 out of 285 in the "antenatal non-growth-restricted" group found to have normal growth after delivery (NPV $=86.31 \%$ ). Therefore, sensitivity and specificity of predicting FGR by serial ultrasound measurements of fetal $\mathrm{HC}$ and fetal AC were 82.59 and 86.62 , respectively. Likelihood ratio of the test is thus 6.2. The pretest probability of a fetus having FGR is $44.1 \%$. Therefore, the probability of having FGR is predicted beyond $80 \%$ by a positive test result. Analysis is given in Table 2 .

Sensitivity $=$ True positives $/[$ True positives + False negatives $]=82.59$ Specificity $=$ True negatives $/[$ True negatives + False positives $]=86.62$ Likelihood ratio $=$ Sensitivity $/ 1-$ Specificity $=6.2$

Out of 508 newborns, 89 (17.5\%) were LBW (birthweight below $2.5 \mathrm{~kg}$ ) newborns. Out of 89 LBW newborns, only 50 (56.2\%) were growth-restricted according to the PI. Remaining 39 (43.8\%) newborns were not growth-restricted. About 419 out of 508 (82.5\%) were of adequate birthweight newborns. Out of that 419 newborns, 175 (41.5\%) were growth-restricted, and 245 (58.5\%) were not growth-restricted (Table 3).

\section{Discussion}

In most of the term IUFD, an underlying undetected FGR is noted. ${ }^{1,5}$ This means many term IUFDs can be prevented if FGR is detected early. Sensitivity of clinical palpation alone to detect SGA, without measuring symphysio-fundal height (SFH) in low-risk populations, is about $20 \%$ and is highly unsatisfactory. ${ }^{2}$

Clinical detection of SGA is also done by relating a spot measurement of SFH to the 10th centile of a growth chart.

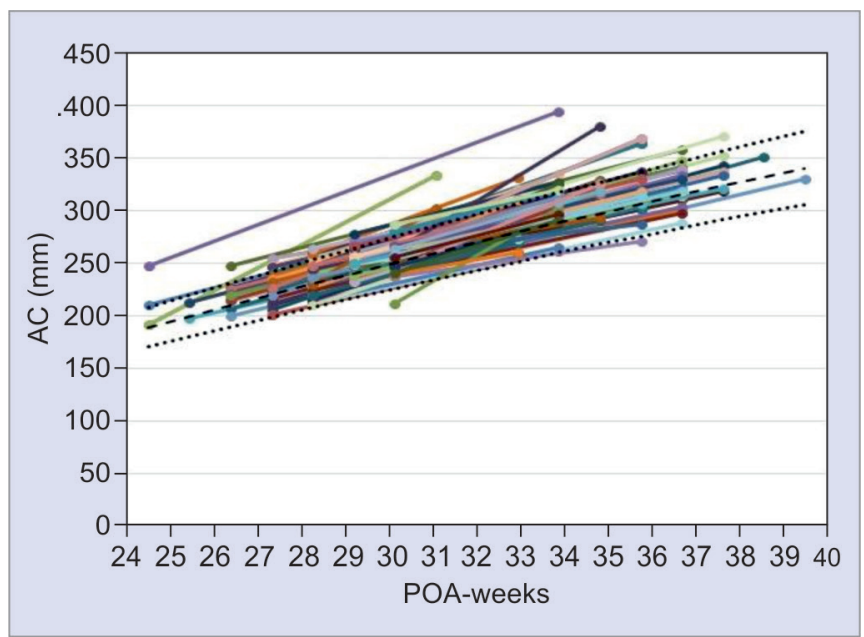

Fig. 4: Distribution of lines connecting two scans of AC in non-growthrestricted fetuses. Dotted lines represent centile of the nomogram

Table 2: Two by two table to calculate sensitivity, specificity, and likelihood ratio

\begin{tabular}{lccc}
\hline & $\begin{array}{c}\text { Confirmed growth- } \\
\text { restricted newborns }\end{array}$ & $\begin{array}{c}\text { Confirmed non-growth- } \\
\text { restricted newborns }\end{array}$ & \\
\hline Antenatal growth-restricted & 185 & 38 & 223 \\
Antenatal non-growth-restricted & 39 & 246 & 285 \\
& 224 & 284 & \\
\hline
\end{tabular}


Table 3: Relationship between LBW and FGR

\begin{tabular}{lrrr}
\hline & FGR & Not FGR & Total \\
\hline LBW $(<2.5 \mathrm{~kg})$ & 50 & 39 & 89 \\
Adequate birthweight $(2.5 \mathrm{~kg})$ & 174 & 245 & 419 \\
Total & 224 & 284 & \\
\hline
\end{tabular}

Naturally, this is not satisfactory due to high inter- and intraobserver variability during measurement as well as the fact that the particular spot measurement, even if above the 10th centile, may well be a FGR. The sensitivity and specificity of detecting SGA by a single measurement of SFH is about 27 and $88 \%$, respectively. ${ }^{2}$ Introduction of a customized SFH chart which is adjusted for maternal characteristics, such as height, weight, parity, and ethnicity, has further improved the detection rate of SGA. Sensitivity of customized SFH chart for detection of SGA is superior to single SFH measurement (48 vs. $27 \%$ ). $2,5,13$

Diagnosis of SGA is based on EFW being below the 10th centile. However, an EFW, despite being above the 10th centile, could well be FGR. Clinical detection of FGR has to be done by detection of the increments in growth with time irrespective of whether the measurements are above or below the 10th centile. Plotting serial SFH on a chart, preferably a customized one, improves the accuracy of this clinical method. ${ }^{2}$ Ultrasound scan is more accurate in detecting FGR. Fetal growth charts have been developed illustrating centile values of fetal $\mathrm{BPD}, \mathrm{HC}, \mathrm{AC}, \mathrm{FL}$, and EFW according to gestational age. Ultrasonically, FGR can be diagnosed when sequential fetal growth measurements show a trajectory deviating to the right of a centile line or cross the centile lines toward the right. Even though fetal growth velocity between two points would be able to detect FGR, there is no clear method of detecting FGR using growth velocity. ${ }^{2}$ Growth velocity differs with the gestational age. In the early part of a gestation, growth velocity is high, whereas in the latter period, it is low, due to physiological senescence of the placenta.

Uterine artery Doppler flow analysis is used in the second trimester to predict FGR while umbilical artery Doppler can diagnose FGR in late pregnancy. RCOG Green-top Guideline recommends offering uterine artery Doppler to pregnant mothers between 20 and 24 weeks, having minor risk factors for FGR. They also recommend serial ultrasound measurement of fetal parameters and umbilical artery Doppler in those who had an abnormal uterine artery Doppler between 20 and 24 weeks. ${ }^{2}$ With advancing FGR, umbilical artery diastolic flow gradually falls, and in late stages, it may be absent or even reversed. Doppler flow analysis can be done in the fetal middle cerebral artery and in the ductus venosus to predict the severity of the FGR. ${ }^{25}$

Antenatally detected SGA, but not FGR, can be verified postnatally by birthweight as all FGR neonates are not SGA. PI has been shown as a tool to identify FGR babies after birth. ${ }^{12,15-23}$ It has been shown that the PI is superior to skin fold thickness, and midarm-circumference-to-occipitofrontal-circumference ratio in detecting growth restricted neonates. ${ }^{12,16}$ It is also known to be better than birthweight centile in detecting growth restriction in neonates. ${ }^{18}$ We used the PI for identification of true growth restriction among neonates.

Thrifty phenotype hypothesis suggests an increased lifetime risk of coronary artery disease, diabetes mellitus, and metabolic syndrome in growth-restricted neonates. ${ }^{6,7}$ The current epidemic of noncommunicable diseases (NCDs) and the inability to improve the outcome of NCDs by late interventions, such as lifestyle modifications and medications, probably lie in the fact that most if not all of these patients with NCDs were programmed in utero during the period of restricted growth. These babies are born with physiological systems with inherently low thresholds for subsequent environmental insults, such as excessive food intake and lack of exercise during postnatal and adolescent life. The inability of the systems to cope with this additional load results in NCDs. Further studies are required to evaluate the outcome of obstetric interventions in mild forms of FGR in preventing NCDs.

Using serial ultrasound measurements of fetal $A C$ and $H C$ and plotting those values on a centile chart is the most accurate way of detecting a growth trajectory. As our results show, sensitivity and specificity of 82.59 and $86.62 \%$, respectively, in the detection of FGR shows that this method enables detection of even mild cases of FGR which cannot be detected by routine methods.

In conclusion, our study has shown that serial ultrasound measurements of fetal $\mathrm{AC}$ and $\mathrm{HC}$ plotted on fetal growth centile charts, with sensitivity, specificity, and a likelihood ratio of 82.9, 86.63 , and 6.2, respectively, provide a more precise method that can detect even milder forms of FGR that are not detected by conventional clinical methods. If ultrasound growth parameter charts and PI centile charts derived from the Sri Lankan population were available, accuracy of antenatal and neonatal confirmation of FGR could have been made even more accurate. Screening low-risk pregnancies for FGR by serial ultrasound scans is the way forward if we are to detect all growth-restricted fetuses and prevent related adult adverse health issues.

\section{Acknowledgments}

The authors acknowledge the support given by staff of the Obstetrics and Gynecology Unit of Sri Jayawardenepura General Hospital.

\section{Author Contributions}

HP designed and planned the study, and supervised data collection and analysis; RD performed ultrasound scans, collected data, and analyzed and drafted the article. Both authors reviewed the article.

\section{References}

1. Lawn JE, Cousens S, Zupan J. 4 million neonatal deaths: When? Where? Why? Why? Lancet 2005;365(9462):891-900. DOI: 10.1016/ S0140-6736(05)71048-5.

2. Green-top guideline No 31. The investigation and management of small for gestational age fetus. 2nd ed. 2013.

3. Luesley DM, Baker NP. Obstetrics and gynaecology: an evidencebased text for MRCOG. 2nd ed. 2004. p. 251-259.

4. Ott WJ. Diagnosis of intrauterine growth restriction: comparison of ultrasound parameters. Am J Perinatol 2002;19(3):133-137. DOI: 10.1055/s-2002-25313.

5. Figueras F, Gardosi J. Intrauterine growth restriction: new concepts in antenatal surveillance, diagnosis, and management. Am J Obstet Gynecol 2011;204(4):288-300. DOI: 10.1016/j.ajog.2010.08.055.

6. Hales CN, Barker DJP. The thrifty phenotype hypothesis: type 2 diabetes. Br Med Bull 2001;60:5-20. DOI: 10.1093/bmb/60.1.5.

7. Barker DJP. The malnourished baby and infant: relationship with type 2 diabetes. Br Med Bull 2001;60(1):69-88. DOI: 10.1093/ bmb/60.1.69.

8. Demicheva E, Crispi F. Long term follow up of intrauterine growth restriction: cardiovascular disorders. Fetal Diagn Ther 2014;36(2): 143-153. DOI: 10.1159/000353633. 
9. Cosmi E, Fanelli T, Visentin S, et al. Consequences in infants that were intrauterine growth restricted. J Pregnancy 2011;2011:364381. DOI: 10.1155/2011/364381.

10. Loughna P, Chitty L, Evans T, et al. Fetal size and dating: charts recommended for clinical obstetric practice. Ultrasound 2009;17(3):161-167. DOI: 10.1179/174313409X448543.

11. Papageorghiou A, Ohuma EO, Altman DG, et al. International standards for fetal growth based on serial ultrasound measurements: the Fetal Growth Longitudinal Study of the INTERGROWTH-21st Project. Lancet 2014;384(9946):869-879. DOI: 10.1016/S0140-6736(14)61490-2.

12. Owen P, Ogah J, Bachmann BLM, et al. Prediction of intrauterine growth restriction with customized estimated fetal weight centiles. BJOG 2003;110(4):411-415.

13. Morse K, Williams A, Gardosi J. Fetal growth screening by fundal height measurement. Best Pract Res Clin Obstet Gynaecol 2009;23(6):809-818. DOI: 10.1016/j.bpobgyn.2009.09.004.

14. Dias T, Shanmugaraja V, Ganeshamoorthy P, et al. Birthweight standards - ability of birthweight percentiles in predicting abnormal fetal growth and outcome. Sri Lanka J Obstet Gynaecol 2014;36(4): 85-88. DOI: 10.4038/sljog.v36i4.7729.

15. Onyiriuka AN, Okkolo AA. Small for gestational age, ponderal index and neonatal polycythemia: a study of their association with maternal hypertension among Nigerian women. Ann Afr Med 2005;4(4):154-159.

16. Akram DS, ArifF. Ponderal index of low birth weight babies - a hospital based study. JPMA 2005;55(6):229-231.

17. Vinthilions AM, Lodeiro JG, Fenestein SJ, et al. Value of fetal ponderal index in predicting growth retardation. Obstet Gynecol 1986;67(4):584-588.
18. Fay RA, Dey PL, Saadie CM, et al. Ponderal index: a better definition of the 'at risk' group with intrauterine growth problems than birth-weight for gestational age in term infants. Aust N Z J Obstet Gynecol 1991;31(1):17-19. DOI: 10.1111/j.1479-828x.1991.tb02755.x.

19. Davis DP, Platts P, Pritchard JM, et al. Nutritional status of light-for -date infants at birth and its influence on early postnatal growth. Arch Dis Childhood 1979;54:703-706.

20. Oluwafemi OR, Njokanma FO, Disu EA, et al. Current pattern of Ponderal Indices of term small for gestational age in a population of Nigerian babies. BMC Pediatr 2013;13:110-117. DOI: 10.1186/14712431-13-110.

21. Roje D, Banovic I, Tadin I, et al. Gestational age - the most important factor of neonatal ponderal index. Yonsei Med J 2004;45(2):273-280. DOI: 10.3349/ymj.2004.45.2.273.

22. Nili F, Makipour M, Mobini J. The value of ponderal index as a prognostic factor in predicting complications in term neonates. Med J Islamic Repub Iran 2003;17(3):197-201.

23. Walther FJ, Ramaekers LH. The ponderal index as a measure of the nutritional status at birth and its relation to some aspects of neonatal morbidity. J Perinat Med 1982;10(1):42-47. DOI: 10.1515/ jpme.1982.10.1.42.

24. Landmann E, Reiss I, Gortner L, et al. Ponderal index for discrimination between symmetric and asymmetric growth restriction: percentiles for neonates from 30 weeks to 43 weeks of gestation. J Matern Fetal Neonat Med 2006;19(3):157-160. DOI: 10.1080/14767050600624786.

25. Hoffman C, Galan HL. Assessing the 'at-risk' fetus: Doppler ultrasound. Curr Opin Obstet Gynaecol 2009;21(2):161-166. DOI: 10.1097/ GCO.0b013e3283292468. 